\title{
Determinants of Quality of Life In Patients with Heart Failure
}

\author{
D0 Geavlete ${ }^{1}$, A Pătru ${ }^{1}$, L Antohi ${ }^{1}$, I Kulcsar ${ }^{1}$, R Razvan ${ }^{1}$, E Stoica ${ }^{1}$, M Zaharia ${ }^{2}$ and 0 Chioncel $^{1}$ \\ ${ }^{1}$ Emergency Institute of Cardiovascular Disease, The University of Medicine and Pharmacy, Bucharest, Romania \\ ${ }^{2}$ St. John Emergency Clinical Hospital Bucharest, Romania \\ *Corresponding author: A Pătru, Emergency Institute of Cardiovascular Disease, Bucharest, Romania
}

\section{ARTICLE INFO}

Received: 䶕 January 20, 2020

Published: 蔧 January 28, 2020

Citation: DO Geavlete, A Pătru, L Antohi, I Kulcsar, R Razvan, E Stoica, M Zaharia, O Chioncel. Determinants of Quality of Life In Patients with Heart Failure. Biomed J Sci \& Tech Res 25(1)-2020. BJSTR. MS.ID.004136.

Keywords: Quality of Life; Heart Failure; Risk Stratification; Determining Factors; Pharmacologic Therapy

\section{ABSTRACT}

Heart failure is a life-threatening disease and it has become a priority for the global public health system nowadays, given the fact that in Europe, over $10 \%$ of the people over 65 years old are affected by this rapidly progressive pathology with high morbidity and mortality rates. In developed countries, where life expectancy is continuously increasing, the prevalence of heart failure is directly proportional, therefore the specific treatment aims not only to improve the survival, but also to increase the quality of life, which had become an important issue. This aspect was highlighted by the randomized clinical Trials carried out in the recent years that included quality of life between the major therapeutic goals in patients with heart failure. Therefore, a careful analysis of all the factors that lead to the improvement of the symptoms of heart failure or in contrast, of the precipitating factors that lead to cardiac decompensations, is necessary to define the impact on the quality of life of these patients.

Abbrevations: HF: Heart Failure; HFpEF: HF with Preserved Ejection Fraction; HFrEF: HF with Reduced Ejection Fraction; QoL: Quality of Life; HR-QoL: Health-Related Quality of Life; WHO: World Health Organization; LVEF: Left Ventricular Ejection Fraction; NYHA: New York Heart Association; 6MWT: 6-Minute Walk Test; MLHF: Minnesota Living with Health Failure; KCCQ: Kansas City Cardiomyopathy Questionnaire; COPD: Chronic Obstructive Pulmonary Disease; NSAID's: Nonsteroidal Anti-Inflammatory Drugs; BB: Beta-Blockers; ACE-I: Angiotensin-Converting-Enzyme Inhibitors

\section{Introduction}

Despite the advanced therapeutic arsenal in Heart Failure (HF), this progressive disease, symptomatic in many clinical aspects and potentially fatal when compared to oncologic diseases, remains a public health issue, involving both the patient, the clinician and also the health system. By the nature of its' prolonged evolution, HF affects the quality of life more pronounced compared to other chronic diseases [1]. The purpose of the treatment, whether we refer to HF with Preserved (HFpEF) or Reduced Ejection Fraction (HFrEF), involves not only increasing survival and reducing hospitalization, but also increasing the quality of life by integrating medical, psychological, social, emotional and mental aspects [2]. The concept of Health-Related Quality of Life (HR-QoL) or quality of life (QoL) was introduced in 1980's and accepted with great interest, as such, shortly after, in randomized clinical trials performed in patients with HF, increasing QoL was one of the primary therapeutic outcomes [3].

The concept refers to all the direct and indirect aspects that reflects the health condition, being the expression of the physical and mental state of the patient in his daily life and it is defined by the World Health Organization (WHO) as " the individuals" perception of their position in life in the context of the culture and value systems in which they live, and in relation to their goals, expectations, standards and concerns" [4]. The concept stands by its' multidimensional character and depends on a wide variety of factors (physical condition, economic status, social factors, spiritual factors, psychological or emotional factors)[5]. Quality of life has thus become both an important standard of treatment in HF as well as a prognostic marker, starting from the stage of diagnosis 
up to the advanced stages of HF, often requiring a multidisciplinary approach. Recently, a multicenter study that enrolled 1037 patients with ventricular systolic dysfunction, noted the association between prognostic factors for advanced HF (age, co-morbidities, recent hospitalization and functional class) and QoL decline [6].

Due to the fact that all these factors impact the mortality and rehospitalization rate, by association, the high predictive value of QoL appears to be one of the main clinical objective in heart failure. Interestingly, the parameters commonly used in HF patients risk stratification (Left Ventricular Ejection Fraction (LVEF), renal dysfunction, hemoglobin levels), were not associated with QoL in other important HF studies (eq. SOLVD). This finding underlines the importance of QoL assessment in HF, as the clinical and biological risk stratification is being insufficient for a complete assessment of the patient $[6,7]$. Generic or specific QoL assessment tools have been shown to be effective and correlate with known determinants of diseases' severity such as LVEF, NYHA functional class (New York Heart Association), 6-Minute Walk Test (6MWT) or serum natriuretic peptide level. Of all, the MLHF (The Minnesota Living with Health Failure) and KCCQ (Kansas City Cardiomyopathy Questionnaire) questionnaires are most commonly used in assessing QoL in HF.

Recent data have shown a significant association of the QoL domains tested in patients with HF and the KCCQ score, a score $\leq 60$ correctly identifying patients who need a more complex approach of QoL deficits. A low score predicts frequent HF hospitalizations, decompensated HF, symptoms such as depression, insomnia, anxiety, pain, higher functional class, poor 6MWT, lower glomerular filtration rate and lower LVEF [8]. Therefore, QoL screening is a critical component in HF patients, having the same importance as the therapeutic strategy, but it is often neglected in daily practice.

\section{Quality of Life Determining Factors}

Different essential domains of life define QoL and should be part of the usual evaluation: physical, emotional and social domains. The physical component includes the evaluation of symptoms and physical condition (fatigue, the functional capacity, insomnia dyspnea, difficulties in maintaining the professional activity). The emotional component refers to anxiety, the loss of control over the own life and disease, the feeling of burden to the family, loss of appetite, depression. The social component comprises the patient's perception of maintaining health, self-care capacity and social life [9]. Of all the above listed parameters, there is evidence that physical symptoms and depression, in an interdependent relationship, are the most important predictors of QoL in HF patient [10]. First, the determinants of the physical spectrum are influenced by depression and the NYHA functional class.

Depression on the other hand, affects QoL through two possible mechanisms: psychologically perceived health status (influenced by HF decompensations, readmissions, poor prognosis, debilitating symptoms and inadequate control of treatment, feeling of helplessness) and poor self-care through the presence of HF symptoms [10]. Depression manifests a high prevalence among patients with HF, whether we refer to acute HF or chronic HF (35-60\%)[11]. History of depression has been shown to be a good predictor for the length of hospitalization and postdischarge mortality, possibly due to a direct relationship with patient compliance to the treatment (OPTIMIZE-HF) [1]. In this regard, cognitive therapy and educational programs have shown their benefit in decreasing the severity of depression, anxiety and associated HF symptoms, leading to increased QoL [12]. Selective inhibitors of serotonin reuptake (sertraline, escitalopram) appears to be safe treatments in HF, although strong evidence of efficacy is lacking [13].

However, it remains unclear whether the increased mortality rates of patients with HF and history of depression may be associated with the disease or the use of associated antidepressant medication. Comorbidities (anemia and iron deficiency, Chronic Obstructive Pulmonary Disease (COPD) and asthma), in-hospital readmissions and a higher functional NYHA class negatively affect QoL. Comorbidities and biological fragility contribute to the lack of compliance, the higher rate of hospitalization, institutionalization and finally death. Comorbidities may interfere with the diagnostic process of HF (eq. COPD in the etiology of dyspnea), may aggravate the HF symptoms, may affect the use of HF medication (the use of Angiotensin-Converting-Enzyme Inhibitors (ACE-I) in patients with severe renal dysfunction, Beta-Blockers (BB) with relative contraindication in asthma). The treatment of comorbidities may also cause worsening of HF (Nonsteroidal Anti-Inflammatory Drugs (NSAIDs) in inflammatory diseases, some anti-neoplastic therapies in oncologic patients).

Interactions between HF and comorbidities' medication may lead to lower efficacy of HF treatment, lower drug safety and may facilitate adverse effects (BB in HFrEF and beta-agonists in asthma and COPD) [13]. Furthermore, the level of evidence for HF treatment in patients with comorbidities is limited, as this population is usually excluded from clinical trials, the effectiveness and safety of interventions being thus indeterminate in the presence of these associated pathologies. Adequate treatment of comorbidities (administration of intravenous iron products - ferric carboxymalthosis in case of iron deficiency, correction of anemia, avoidance of medication that aggravates HF, complete decongestion at discharge after hospitalized $\mathrm{HF}$ and recognition of subclinical congestion to avoid frequent decompensations or readmission) promote the increase of QoL in HF patients. Patient's age does not seem to represent a predictor of QoL in patients with HF and literature reports aroused from the current studies are providing contradictory data [14-16].

Erectile dysfunction is another important parameter to consider when it comes to QoL assessment, especially in men. Up 
to $87 \%$ of the HF patients report sexual problems affecting QoL, and their perception is that both symptoms (20\%) and treatment $(10 \%)$ are the main causes. HF treatment may interfere with sexual activity (thiazide diuretics, spironolactone, beta-blockers). Phosphodiesterase inhibitors, which through their beneficial hemodynamic and anti-vascular remodeling effects may increase exercise capacity and QoL, are useful and safe, but should not be used in combination with other medication (nitrates) [13]. Affecting the QoL in relation to the sexual activity resides also from the poor management of this particular issue, by not addressing the real problems in the cardiac rehabilitation programs or during the scheduled visits, the consequences being important for the patient (anxiety, depression, non-adherence to treatment, self-medication that can interfere with the HF treatment or social problems) and in direct relation with long-term prognosis.

\section{Discussion}

It is important to mention that besides the analysis upon the determinant factors that impact the quality of life, the role of pharmacological and non- pharmacological therapy in increasing the quality of life is crucial for heart failure patients.

\section{The Role of Pharmacological Therapy in Increasing the QoL in Heart Failure Patients}

Consistent evidence for increasing QoL for major HFspecific therapeutic classes is limited. Literature data show the improvement of QoL when using angiotensin receptor blockers (CHARM and Val-HeFT trial), ACE-I, ivabradine (SHIFT trial), the combination of hydralazine - isosorbide dinitrate (A-HeFT trial), sacubitril-valsartan (PARADIGM-HF trial), device therapy (COMPANION, CARE-HF trials) or cardiac rehabilitation $[13,17,18]$. For example, beta-blockers in HF, although significantly improve LV function, do not have a consistent QoL enhancement effect, but there are data from observational studies that mention a beneficial effect of bisoprol in HF patients QoL enhancement [19]. In advanced stages of the disease, in advanced HF, palliative medication such as positive inotropic agents and vasoactive therapy becomes the main priority. In these cases, QoL assessment differs, the purpose of the treatment being different: survival becomes the main objective of the treatment in the acute phase as the mortality is extremely high the QoL standard downgrading to secondary level. Study evidence in the acute phase of the disease, related to the specific medication (dobutamine, milrinone, levosimendan, nitroprusiat, nesiritide) is limited, but there are reports showing favorable effects and impact on QoL parameters for levosimendan alone, probably due to the effect on secondary organ dysfunction by improving right ventricular dysfunction [1].

\section{The Role of Non-Pharmacological Therapy in Improving the QoL in Heart Failure Patients}

Physical activity plays a central role in the treatment recommendations of HF as an important component of the therapy, regardless of LVEF, leading to the improvement of the effort capacity and the QoL [20]. Device therapy (cardiac resynchronization, mitral clips and modulation of cardiac contractility in selected patients) has also shown its' benefit upon QoL improvement [13]. Cardiac resynchronization therapy (CRT) contributes to increased QoL through symptoms improvement and cardiac performance in patients with moderate-severe HF (NYHA class III / IV) [13]. As for the implantable cardiac defibrillator, recent data recognize an negative impact on QoL, through anxiety and frequent hospitalizations associated with the administration of internal electric shocks in advanced stages of the disease. Thus, in selected cases with advanced $\mathrm{HF}$, it might be worth reconsidering the benefit of measures leading to increased survival when they severely affect QoL, taking into consideration the patient's options $[1,20]$. And this idea is sustained by the fact that in patient's perspective, satisfactory survival is closely linked to an adequate QoL, and not rarely the patients prioritize QoL even at the cost of reducing their lifespan.

\section{Conclusions}

QoL assessment in heart failure patients has lately become as important as the treatment itself, combining both therapeutic and prognostic value for the patients. But due to the complexity of $\mathrm{HF}$ patients care, limited time and resources as well as the absence of specific tools for assessing QoL, this evaluation is unfortunately often neglected in clinical practice. The clinical utility of questionnaires and tools for assessing the QoL, the impact of QoL deficits treatment on prognosis and objectives in HF (survival and rehospitalization) and the resources needed for screening and treating them are some important issues to be answered. And these questions should be addressed in clinical studies, in representative populations of patients with HF in order to establish recommendations supported by clinical evidence.

\section{References}

1. Nieminen MS, Dickstein K, Fonseca C, Serrano JM, Parissis J, et al. (2015) The patient perspective: Quality of life in advanced heart failure with frequent hospitalisations. Int J Cardiol 191: 256-264.

2. Jaarsma T, Beattie JM, Ryder M, Rutten FH, McDonagh T et al. (2009) Palliative care in heart failure: a position statement from the palliative care workshop of the Heart Failure Association of the European Society of Cardiology. EJHF 11(5): 433-443.

3. Dunderdale K, Thompson DR, Miles JN, Beer SF, Furze G (2005) Qualityof-life measurement in chronic heart failure: do we take account of the patient perspective? Eur J Heart Fail 7(4): 572-582.

4. (1995) The World Health Organization Quality of Life assessment (WHOQOL): position paper from the World Health Organization. Soc Sci Med 41(10): 1403-1409.

5. MacIver J, Wentlandt K, Ross HJ (2017) Measuring quality of life in advanced heart failure. Curr Opin Support Palliat Care 11(1): 12-16.

6. Comin Colet J, Anguita M, Formiga F, Almenar L, Crespo Leiro MG, et al. (2016) Health-related Quality of Life of Patients With Chronic Systolic Heart Failure in Spain: Results of the VIDA-IC Study. Rev Esp Cardiol 69(3): 256-271. 
7. Gorkin L, Norvell NK, Rosen RC, Charles E, Shumaker SA, et al. (1993) Assessment of quality of life as observed from the baseline data of the Studies of Left Ventricular Dysfunction (SOLVD) trial quality-of-life substudy. AmJCardiol 71(12): 1069-1073.

8. Cavanagh CE (2019) Quality of life in heart failure: screening alone is insufficient. Eur Heart J Qual Care Clin Outcomes 5(3): 191-192.

9. Hwang SL, Liao WC, Huang TY (2014) Predictors of quality of life in patients with heart failure. Jpn J Nurs Sci 11(4): 290-298.

10. G Gnanasekaran (2011) Epidemiology of depression in heart failure. Heart Fail Clin 7(1): 1-10.

11. Blumenthal JA, Babyak MA, O Connor C, Keteyian S, Landzberg J, et al. (2012) Effects of exercise train-ing on depressive symptoms in patients with chronic heart failure: the HF-ACTION randomized trial. JAMA 308(5): 465-474.

12. Ponikowski P, Voors AA, Anker SD, Bueno H, Cleland JGF, et al. (2016) 2016 ESC Guidelines for the diagnosis and treatment of acute and chronic heart failure. European Heart Journal 37(27): 2129-2200.

13. Zambroski CH, Moser DK, Bhat G, Ziegler C (2005) Impact of symptom prevalence and symptom burden onquality of life in patients with heart failure. EuropeanJournal of Cardiovascular Nursing 5: 198-206.

14. Heo S, Moser DK, Riegel B, Hall LA, Christman N (2005) Testing the psychometric properties of the Minnesota Living with Heart Failure questionnaire. Nursing Research 54(4): 265-272.

\section{ISSN: 2574-1241}

DOI: $10.26717 /$ BJSTR.2020.25.004136

A Pătru. Biomed J Sci \& Tech Res

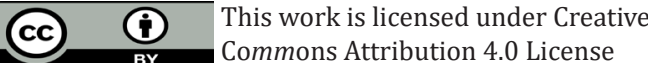

Submission Link: https://biomedres.us/submit-manuscript.php
15. Van der Wal HH, van Deursen VM, van der Meer P, Voors AA (2017) Comorbidities in Heart Failure. Handb Exp Pharmacol 243: 35-66.

16. McMurray JJ, Adamopoulos S, Anker SD, Auricchio A, Böhm M, et al. (2012) ESC Guidelines for the diagnosis and treatment of acute and chronic heart failure 2012: The Task Force for the Diagnosis and Treatment of Acute and Chronic Heart Failure 2012 of the European Society of Cardiology. Eur Heart J 33(14): 1787-1847.

17. Chandra A, Lewis EF, Claggett BL, Desai AS, Packer M, et al. (2018) Effects of Sacubitril/Valsartan on Physical and Social Activity Limitations in Patients With Heart Failure: A Secondary Analysis of the PARADIGM-HF Trial. JAMA Cardiol 3(6): 498-505.

18. González Juanatey JR, Alegría Ezquerra E, García Saavedra V (2003) Use of bisoprolol in heart failure. The BISOCOR observational study. Rev Esp Cardiol 56(9): 873-879.

19. Fukuta H, Goto T, Wakami K, Ohte N (2016) Effects of drug and exercise intervention on functional capacity and quality of life in heart failure with preserved ejection fraction: A meta-analysis of randomized controlled trials. Eur J Prev Cardiol 23(1): 78-85.

20. Flint K, Fairclough DL, Spertus JA, Bekelman DB (2019) Does heart failure-specific health status identify patients with bothersome symptoms, depression, anxiety, and/or poorer spiritual well-being? Eur Heart J Qual Care Clin Outcomes 5(3): 233-241.

$\begin{array}{ll}\text { BIOMEDICAL } & \text { Assets of Publishing with us } \\ \text { RESEARCHES } & \text { - Global archiving of articles } \\ \text { - Immediate, unrestricted online access }\end{array}$

